\title{
Pervasive and Intelligent Decision Support in Intensive Medicine - The Complete Picture
}

Filipe Portela ${ }^{1}$, Manuel Filipe Satos ${ }^{1}$, José Machado, António Abelha ${ }^{2}$, Álvaro Silva ${ }^{3}$, and Fernando Rua ${ }^{3}$

${ }^{1}$ Algoritmi Centre, University of Minho, Portugal \{cfp, mfs\}@dsi.uminho.pt

${ }^{2}$ CCTC, University of Minho, Portugal

\{jmac, abelha\}edi.uminho.pt

${ }^{3}$ Serviços de Cuidados Intensivos, Centro Hospitalar do Porto, Hospital Santo António, Portugal

moreirasilva@gmail.com, fernadorua.sci@hgsa.min-saude.pt

\begin{abstract}
In the Intensive Care Units (ICU) it is notorious the high number of data sources available. This situation brings more complexity to the way of how a professional makes a decision based on information provided by those data sources. Normally, the decisions are based on empirical knowledge and common sense. Often, they don't make use of the information provided by the ICU data sources, due to the difficulty in understanding them. To overcome these constraints an integrated and pervasive system called INTCare has been deployed. This paper is focused in presenting the system architecture and the knowledge obtained by each one of the decision modules: Patient Vital Signs, Critical Events, ICU Medical Scores and Ensemble Data Mining. This system is able to make hourly predictions in terms of organ failure and outcome. High values of sensitivity where reached, e.g. $97.95 \%$ for the cardiovascular system, $99.77 \%$ for the outcome. In addition, the system is prepared for tracking patients' critical events and for evaluating medical scores automatically and in real time.
\end{abstract}

\section{Introduction}

Nowadays, it is recognized that the Intensive Care Units (ICU) are filled with many technical devices for monitoring their patients. However it is also recognized by ICU professionals that normally the data are stored into the patient records and rarely are used to support the decision process. After some studies it was possible to conclude that using these data in a correct way, it is possible to take some advantages in order to support the Decision Making Process (DMP). As the main goal was to support the decision making process in a pervasive way and using intelligent systems, the first task that had to be carried out was change the environment in order to use the medical devices and to collect the patient data automatically and in real-time. Then, using the data provided by the environment combined with automatic tasks of data transforming it was necessary prepare the data according to some variables used by the system in order to pursuit new knowledge.

This process became a reality with the adoption of INTCare system in the ICU of Centro Hospitalar do Porto (CHP), Porto, Portugal. INTCare is a pervasive intelligent decision support system composed by a set of integrated modules which performs all the tasks of Knowledge Discovery in Database process automatically and in real-time. INTCare can present anywhere and anytime information / Knowledge essential for DMP. INTCare can provide:

a) Patient Clinical data (Vital Signs, Fluid Balance, Patient Scales Laboratory Results); 
b) Critical Events tracking (SPo2, Heart Rate, Blood Pressure, Urine Output and Temperature);

c) ICU Medical Scores (SAPS II, SAPS III, Glasgow SOFA, MEWS and TISS);

d) Probability related to Organ Failure (Cardiovascular, Coagulation, Respiratory, Hepatic and Renal) and to patient discharge condition (live or death).

The main goal of the project is integrating a set of data sources of data sources and taking advantages of the interoperability and the use of Data Mining in order to develop a set of pre-defined functions to produce new knowledge important to the DMP. As solution it was deployed a pervasive intelligent framework that operates in real-time, anywhere and anytime. This paper presents the ICU information system architecture developed, the integrated platform to support DMP and the main knowledge attained. This paper is divided into six sections. The first section introduces the paper and INTCare system as well the knowledge obtained. The second section presents the project background and introduces a set of concepts associated to the work. The third section makes an overview on the information system architecture and the Intelligent Decision Support System. The section four presents the modifications introduced into the ICU and to DMP for pervasive decision supporting. Finally, section five presents the results achieved related to the creation of knowledge and, in the sixth section, some conclusion remarks close the paper.

\section{Background}

\subsection{Intensive Care Units}

The Intensive Care Units (ICU) is a particular unit where a special area of medicine is applied: Intensive Medicine (IM). The goal of IM is recover the patient in a serious health condition to a previous state, i.e., the condition verified before the ICU admission [1]. ICU is characterized to be a critical environment where the patient is normally in coma and he is constantly monitored. At same time he is always the first concern. In these cases when something happen in the ICU with the patient, the decision need to be quickly performed. Daily, ICU professionals are dealing with human lives and a good decision is fundamental to save their lives. In this point the possibility of having some extra knowledge to support the decision process in realtime it is very important. Normally in the ICUs the patient documentation is done manually, offline and the data is stored in a paper format [2].

INTCare changed this paradigm and improved the method how the data was collected from manual actions made by ICU professionals to a totally automatic and real-time process that avoids the paper records.

\subsection{INTCare}

INTCare is a research project to intensive medicine. The first goal of INTCare was to develop an intelligent system to predict the organ failure and patient outcome [3]. In 2009 it was notorious in the CHP the high numbers of data stored manually, in the 
paper format and associated a set of barriers which implied the non-execution of the project. After make a set of studies was possible define which were the ICU information system gaps $[4,5]$ and to present a new solution to the service.

The solution adopted was based in intelligent agents [6]. These agents performs some tasks automatically as is, data acquisition and data processing, at same time the agents are responsible to prepared the system to be deployed in pervasive environments [7]. As result, INTCare is now a Pervasive Intelligent Decision Support System (PIDSS) that acts automatically and in real-time in order to give new information (knowledge) to the ICU decision makers (physicians and nurses).

\subsection{Pervasive Decision Making Process}

The Decision Making Process (DMP) of ICU is a key for saving lives. To the physicians it is very important having the patient information in the right time. According to some studies, the medical error is the eighth leading cause of death in industrialized countries [8]. The DMP can evolve to a different way of taking decisions. Taking into account the pervasive health care: "conceptual system of providing healthcare to anyone, at any time, and anywhere by removing restraints of time and location while increasing both the coverage and the quality of healthcare" [9], it is possible explore some contexts in order to develop pervasive systems.

The development of a PIDSS that helps the decision process giving the right information in the right moment can improve the DMP. A PIDSS can fill most of the gaps and help the coordination of several activities that can be as important to the survival of the patient as determine the correct diagnosis and execute the appropriate procedures [10].

\subsection{Critical Events}

Studies done in the past reported that the most common critical errors were due to wrong mechanical or human performance [11]. Before the project start the clinical critical events weren't considered in this unit. The critical events are now in use and are assigned in an electronic application at a continuous acquisition basis. To understand if an event it is critic, two main criteria were used [1]:

- Occurrence and duration should be registered by physiological changes;

- Related physiological variables should be routinely registered at regular intervals.

An event is considered critical, when a longer event occurs or a more extreme physiologic measurement is found [1].

\subsection{ICU Medical Scores}

Medical Scores are integrated in the diagnosis-related groups [12] and can be used, for example, to predict the outcome [13]. In the ICU of CHP, the most common scores are: SOFA, SAPS II and Glasgow. To help the DMP two more clinical scores were added: TISS 28 and MEWS. 
Sepsis-related Organ Failure Assessment (SOFA) is used to daily score, as objectively as possible, the degree of organ dysfunction/failure of a patient [14].

Simplified Acute Physiology Score II (SAPS II) is an evolution of SAPS and provides an estimation of the risk of death without having to specify a primary diagnosis.

Glasgow Coma Score (GCS) [15] describes the patient's level of consciousness.

Therapeutic Intervention Scoring System (TISS-28), quantifies type and number of intensive care treatments [16].

Modified Early Warning Score (MEWS) [17] is a track and trigger scoring system that is used to monitor changes in a patient's physiology.

\subsection{Ensemble Data Mining}

In this project and in order to induce Ensemble Data Mining models it is used the data streaming. According to Gaber [18], the Data Stream Mining (DSM) is concerned with extracting knowledge structures represented in models and patterns and in nonstopping streams of information. The ensemble-learning methodology consists in two sequential phases: the training and the testing phase [19]. In the training phase, several different predictive models are generated from the training set. In the test phase, the ensemble is executed and aggregates the outputs for each predictive model [19].

\subsection{Research Methodologies}

All of this work is a result of a research project. In order to obtain good results, a set of research methodologies were used. The main methodology used it was design research. Design Research (DR) is the main research of this work, i.e., DR drove the entire project, because it is fundamental in the developing of effective solutions.

Design research is fundamental to creating products, services, and systems that respond to human needs [20]. According Lee, P [20], DR has as primary goal generate value for the end user and as result develop a sound solution that meets identified needs.

According Lunenfeld [21] research for design is the hardest to characterize, as its purpose is to create objects and systems that display the results of the research and prove its worth. One of the main goals of DR is to understand and improve the design processes and practices quite sketchily. This represents more than developing a specific knowledge domain in a professional field because encloses the environment and their stakeholders. INTCare followed the DR methodology, because the solution developed meets the ICU professional's needs and the generated knowledge is fundamental to support the DMP. 


\section{Information System}

\subsection{Data Acquisition}

\section{a) Architecture}

The data used by the system was obtained using two ways of acquisition: manually and automatically. Initially many of the data were acquired manually and recorded in the paper nursing records.

Nowadays the scenario is different, the data is automatically collected and stored in the database recurring to the use of intelligent agents and automatic procedures.

As Figure 1 shows the data is acquired in real-time and in an electronic format using automatic or manual procedures. Finally the data acquired is stored in the database and available online through the Electronic Nursing Record (ENR).

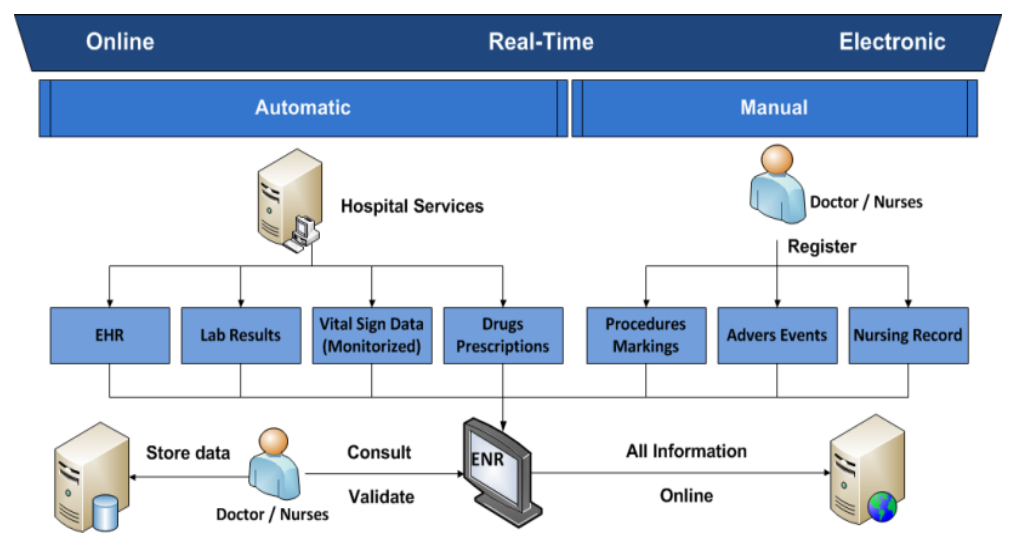

Fig. 1. Data Acquisition Arquitecture

\section{b) Data Overview}

This data is provided from several data sources:

- $\quad$ Bedside Monitors (BM);

- $\quad$ Electronic Nursing Record (ENR);

- $\quad$ Electronic Health Record (EHR);

- Laboratory (LAB);

- Drugs System. (DS).

Table 1 present the data collected and which data is used by the attributes of each one of the decision sub-systems:

- Critical Events (CE);

- Vital Signs (VS);

- $\quad$ Scoring System (SS);

- $\quad$ Ensemble Data Mining (EDM).

All the data is stored in real-time, i.e., in the moment of the value is collected. 
Table 1. Data Sources

\begin{tabular}{|c|c|c|c|c|c|}
\hline Variables & Data Source & $\mathbf{C E}$ & VS & SS & EDM \\
\hline Blood Pressure & $\mathrm{BM} / \mathrm{ENR}$ & $\mathrm{X}$ & $\mathrm{X}$ & $\mathrm{X}$ & $\mathrm{X}$ \\
\hline Heart Rate & $\mathrm{BM} / \mathrm{ENR}$ & $\mathrm{X}$ & $\mathrm{X}$ & $\mathrm{X}$ & $\mathrm{X}$ \\
\hline Respiratory Rate & $\mathrm{BM} / \mathrm{ENR}$ & & $\mathrm{X}$ & $\mathrm{X}$ & \\
\hline Saturation of Oxygen ( $\mathrm{SpO} 2)$ & $\mathrm{BM} / \mathrm{ENR}$ & $\mathrm{X}$ & $\mathrm{X}$ & $\mathrm{X}$ & $\mathrm{X}$ \\
\hline Temperature & $\mathrm{BM} / \mathrm{ENR}$ & $\mathrm{X}$ & $\mathrm{X}$ & $\mathrm{X}$ & $\mathrm{X}$ \\
\hline Vasopressors & DS & & & $\mathrm{X}$ & $\mathrm{X}$ \\
\hline Admission and Discharge data & EHR & & & $\mathrm{X}$ & $\mathrm{X}$ \\
\hline AGE & EHR & & & $\mathrm{X}$ & $\mathrm{X}$ \\
\hline Chronic diseases & EHR & & & $\mathrm{X}$ & \\
\hline Clinical Events & EHR & & & $\mathrm{X}$ & \\
\hline Clinical Procedures & EHR & & & $\mathrm{X}$ & \\
\hline AVPU & ENR & & & $\mathrm{X}$ & \\
\hline Glasgow & ENR & & & $\mathrm{X}$ & $\mathrm{X}$ \\
\hline Urine Output / Diuresis & ENR & $\mathrm{X}$ & & $\mathrm{X}$ & $\mathrm{X}$ \\
\hline Albumin and BUN & LAB & & & $\mathrm{X}$ & \\
\hline Bilirubin / Creatinine & LAB & & & $\mathrm{X}$ & $\mathrm{X}$ \\
\hline $\mathrm{FiO} 2$ and $\mathrm{PaO} 2 / \mathrm{WBC}$ & LAB & & & $\mathrm{X}$ & $\mathrm{X}$ \\
\hline Glucose / HCO3/ Leucocytes / PH & LAB & & & $\mathrm{X}$ & \\
\hline Platelets / Potassium / Sodium/ Urea & LAB & & & $\mathrm{X}$ & \\
\hline
\end{tabular}

\subsection{Intelligent Decision Support System}

The Intelligent Decision Support System (IDSS) architecture involves a set of systems and changes in the ICU environments. This IDSS it is characterized by attending some requirements [7, 22]: Online-Learning, Real-Time, Adaptability, Data mining models, Optimization, Intelligent agents, Accuracy Safety, Pervasive / Ubiquitous, Privacy, Secure Access from Exterior, User Policy, integration and interoperability.

Figure 2 presents the IDSS architecture in order to produce knowledge and demonstrates an overview of the IDSS process as a whole. First, the data is acquired from five data sources (Bedside Monitors, Laboratory, Drugs System, Electronic Nursing Record and Electronic Health Record), Then the data are validate and preprocessed according to have a correct patient identification and the values are real, i.e., the data are between the possible range defined by ICU [7]. After the data to be stored in database they are transformed according the IDSS target. Each variable is prepared to be an input attribute of the inference engine. Finally the result is produced in the inference engine, through the use of ensembles data mining and automatic data processing.

The results are presented by INTCare and ENR platform. In the other side of the process, they are the ICU professionals that can consult all the knowledge produced by the inference system anywhere and anytime using for the effect a mobile device with internet access. By using remote access, ICU professionals can consult the values of the vital signs system, scoring system and critical events system and the prevision of patient condition for several variables. The system is recognized by having some particular characteristics of pervasive computing [23]: scalability, heterogeneity, integration, invisibility and Context awareness. 


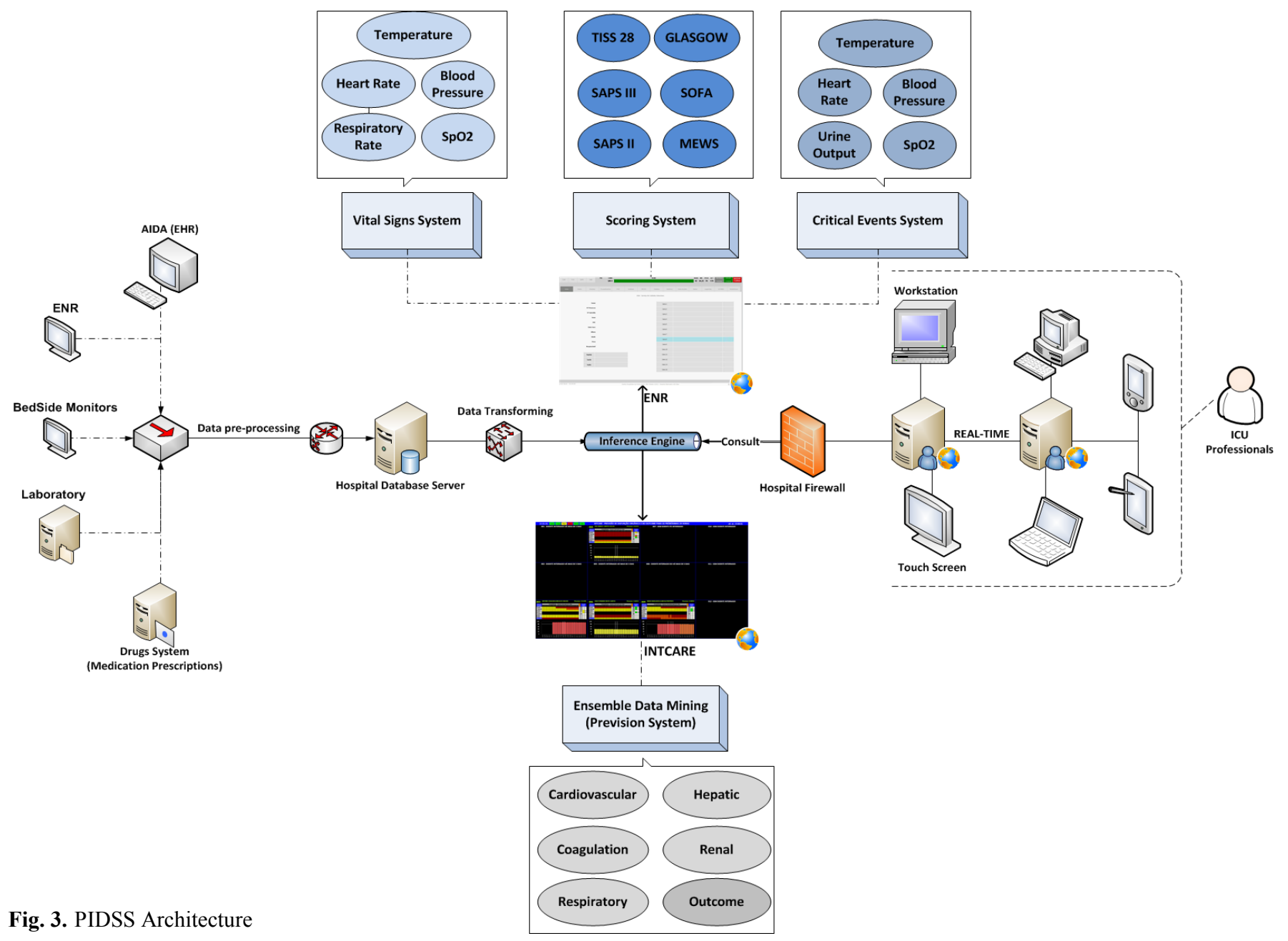

\section{Pervasive Decision Support}

In order to improve the Decision Making Process (DMP) a set of new knowledge was obtained using the changes deployed in the Information System in order to make the DMP a pervasive process. In this context the way of how the patient clinical data are acquired was modified, and four intelligent systems were deployed.

\subsection{Patient Clinical Data}

With the introduction of the changes in the Information System a set of patient clinical data became available in INTCare platforms to be consulted and to help the 
decision making process. Table 2 presents which data is available electronically, online and in real-time (ORT) and if the user can consult, edit or validate the values.

Table 2. Information available in ICU

\begin{tabular}{|c|c|c|c|c|}
\hline Information Type & ORT & Consult & Edit & Validate \\
\hline Admission and Dischage data & $\sqrt{ }$ & $\sqrt{ }$ & & \\
\hline Clinical Interventions & $\sqrt{ }$ & $\sqrt{ }$ & - & - \\
\hline Clinical Procedures & $\sqrt{ }$ & $\sqrt{ }$ & - & - \\
\hline Diagnostics tests & $\sqrt{ }$ & $\sqrt{ }$ & - & - \\
\hline Eletronic Health Record & $\sqrt{ }$ & $\sqrt{ }$ & $\sqrt{ }$ & $\sqrt{ }$ \\
\hline Fluid Balance & $\sqrt{ }$ & $\sqrt{ }$ & $\sqrt{ }$ & $\sqrt{ }$ \\
\hline Lab Results & $\sqrt{ }$ & $\sqrt{ }$ & - & - \\
\hline Medical Requests & $\sqrt{ }$ & $\sqrt{ }$ & - & - \\
\hline Medical Scores & $\sqrt{ }$ & $\sqrt{ }$ & $\sqrt{ }$ & $\sqrt{ }$ \\
\hline Nursing Notes & $\sqrt{ }$ & $\sqrt{ }$ & $\sqrt{ }$ & $\sqrt{ }$ \\
\hline Other Records & $\sqrt{ }$ & $\sqrt{ }$ & $\sqrt{ }$ & $\sqrt{ }$ \\
\hline Pain Scales & $\sqrt{ }$ & $\sqrt{ }$ & $\sqrt{ }$ & $\sqrt{ }$ \\
\hline Patient Information & $\sqrt{ }$ & $\sqrt{ }$ & $\sqrt{ }$ & $\sqrt{ }$ \\
\hline Patient Procedures & $\sqrt{ }$ & $\sqrt{ }$ & $\sqrt{ }$ & $\sqrt{ }$ \\
\hline Prescription Plan & $\sqrt{ }$ & $\sqrt{ }$ & - & $\sqrt{ }$ \\
\hline Therapeutic Attitudes & $\sqrt{ }$ & $\sqrt{ }$ & $\sqrt{ }$ & $\sqrt{ }$ \\
\hline Therapeutic Plan & $\sqrt{ }$ & $\sqrt{ }$ & $\sqrt{ }$ & $\sqrt{ }$ \\
\hline Ventilation & $\sqrt{ }$ & $\sqrt{ }$ & $\sqrt{ }$ & $\sqrt{ }$ \\
\hline Vital Signs & $\sqrt{ }$ & $\sqrt{ }$ & $\sqrt{ }$ & $\sqrt{ }$ \\
\hline \multicolumn{5}{|c|}{ Knowledge } \\
\hline Chart - MEWS & $\sqrt{ }$ & $\sqrt{ }$ & & \\
\hline Chart - Scores & $\sqrt{ }$ & $\sqrt{ }$ & & \\
\hline Chart - TISS 28 & $\sqrt{ }$ & $\sqrt{ }$ & & \\
\hline Chart - Critical Event & $\sqrt{ }$ & $\sqrt{ }$ & & \\
\hline Chart - Vital Signs & $\sqrt{ }$ & $\sqrt{ }$ & & \\
\hline Critical Events & $\sqrt{ }$ & $\sqrt{ }$ & & \\
\hline Medical Scores & $\sqrt{ }$ & $\sqrt{ }$ & & \\
\hline Probability of Organ Failure & $\sqrt{ }$ & $\sqrt{ }$ & & \\
\hline Probability of Patient Die & $\sqrt{ }$ & $\sqrt{ }$ & & \\
\hline
\end{tabular}

\subsection{Critical Events}

In this project two different definitions are used: critical values and critical events. Critical values are values that are out of a normal range during an undefined time. Critical event is defined as a label to identify that a variable had critical values for more than the admissible time span (type a), as defined in Table 3. An event also can be considered critical when the value was too much out of the normal range (considered serious) regardless of the duration of that observation (type b). For example, a critical event happens whenever the patient's blood pressure stays above $180 \mathrm{mmHg}$ or below $90 \mathrm{mmHg}$ for more than 1 hour. Also, a critical event happens every time the blood pressure drops below $60 \mathrm{mmHg}$. 
Table 3. The protocol for the out of range measurements (adapted from [1] )

\begin{tabular}{crrrrr}
\hline & $\begin{array}{c}\text { BP } \\
\text { (mmHg) }\end{array}$ & $\begin{array}{c}\text { SpO2 } \\
\mathbf{( \% )}\end{array}$ & \multicolumn{1}{c}{$\begin{array}{c}\text { HR } \\
\text { (bpm) }\end{array}$} & $\begin{array}{c}\text { UR } \\
(\mathbf{m l} / \mathbf{h})\end{array}$ & \multicolumn{1}{c}{$\begin{array}{c}\text { Temp. } \\
\left({ }^{\circ} \mathbf{C}\right)\end{array}$} \\
\hline Normal range & 90 to 180 & $>=90$ & 60 to 120 & $>=30$ & 35 to 37 \\
Critical event $\mathbf{a}$ & $>=1 \mathrm{~h}$ & $>=1 \mathrm{~h}$ & $>=1 \mathrm{~h}$ & $>=2 \mathrm{~h}$ & $>=1 \mathrm{~h}$ \\
Critical event $\mathbf{b}$ & $<60$ & $<80$ & $<30>180$ & $<=10$ & $<34>41$ \\
\hline
\end{tabular}

a Defined when continuously out of range.

b Defined anytime.

The tracking system is executed in real-time using the automatic data acquisition and data processing tasks.

\subsection{ICU Medical Scores}

The scoring system (SS) was developed in order to introduce a new concept of calculating scores. Instead of the scores being calculated at the end of the day, the system can calculate in real-time some ICU scores.

SS uses the processing and transformation rules defined for each score, to automatically and in real-time acquire and processing the data in order to obtain: SAPS II, SAPS III, GLASGOW, SOFA, TISS 28 and MEWS. Despite of many of the data are collected automatically some of them require human observation and consequence manual store (eg. Glasgow, Urine Output).

The SS is integrated in the Electronic Nursing Record (ENR). The ICU staff can consult the results through this application. This application is also used for registering some values that require a human observation like is Glasgow and some SAPS parameters. The scores results are calculated automatically and in real-time whenever a new value arrives. The SS has always in consideration the worst value collected.

\subsection{Prediction Models}

The prediction models were constructed using the characteristics of ensemble data mining. The ensemble is organized in terms of six independent components. Each one of these components is dedicated to a different target (Renal, Respiratory, Hepatic, Coagulation, Cardiovascular or Outcome), considers seven different scenarios (S1 to S7) and applies three distinct DM techniques: Decision Trees (DT), Support Vector Machine (SVM) and Naïve Bayes (NB).

The ensemble can be defined as a three-dimensional matrix $M$ composed by $s=7$ scenarios ( $s 1$ to $s 7) \times t=6$ targets ( $\mathrm{t} 1$ to $\mathrm{t} 7) \mathrm{x} \mathrm{z}=3$ techniques ( $\mathrm{z} 1$ to $\mathrm{z} 3$ ). Each element of $M$ corresponds to a particular model and can be defined as:

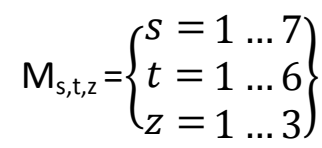




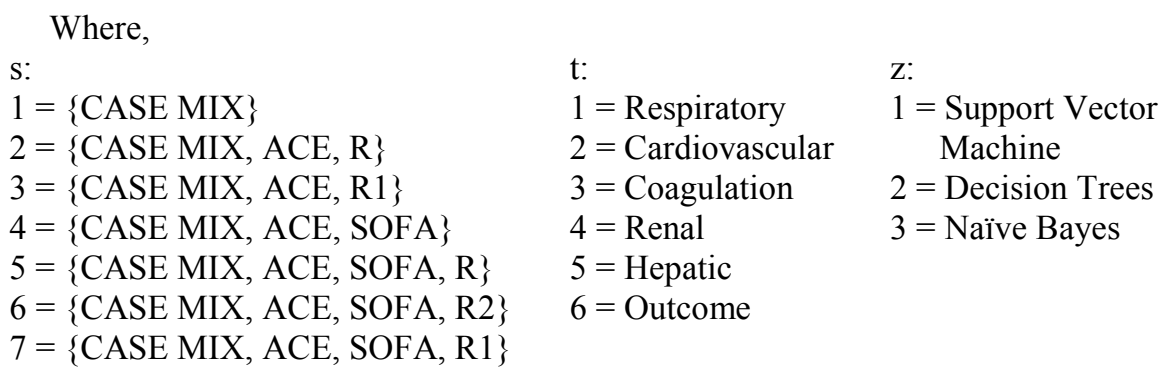

Each model was induced automatically and in real-time using streamed data. The data mining engine uses the data present in input table of the patient admitted in ICU. Then the models are induced using online-learning by the DM agent. This agent runs whenever a request is sent or when the performance of the models decreases. The ensemble process is composed by:

- Predictive Models - 126 models are induced combining seven scenarios (S1 to S7), six targets and three different techniques (SVM, DT and NB);

- Ensemble - the models are assessed in terms of the sensitivity, accuracy, total error and specificity. The best model for each target $(\mathrm{t})$ is then selected.

In order to choose the best predictive model for each target a set of tasks are performed automatically and in real-time:

1. Create the confusion matrix for each scenario;

2. Obtain the assessment measures;

3. Apply the quality measure;

4. Determine the confidence rate for each prediction.

\section{RESULTS}

At level of results it was possible dematerialize the ICU processes, making the patient clinical data that earlier were manually collected and stored in the paper, available electronically. As figure 2 showed the pervasive IDSS is composed by four modules:

$\checkmark$ Vital signs;

$\checkmark$ Medical Scores;

$\checkmark$ Critical events;

$\checkmark$ Ensemble data mining.

\section{$5.1 \quad$ Vital Signs}

For the vital signs it is possible consult all the data collected by the system and observe the evolution of vital signs values from a patient. The results are present in two forms: a grid and a chart. The grid has 24 columns, one for each hour and 11 lines, one for each vital sign type (Blood Pressure (BP) - Diastolic and Systolic, Mean Blood Pressure (MBP), Intracranial pressure (ICP), Central Venous Pressure (CVP), Respiratory Rate (RR), Heart Rate (HR), Non-Invasive Blood Pressure 
(NIBP) - Diastolic and Systolic, Saturation of Oxygen (SpO2) and Temperature. The grid is filled automatically at long of day with the first value collected for each hour / vital sign. The chart presents a graphic analysis of the Vital Signs Evolution for BP, HR, RR, CVP, SpO2 and Temperature. This chart has three types of visualization (Minute, Hour and Day). The first chart presents the values collected by minute, the hour chart (figure 3 ) presents an average of all values collected by hour and the day chart presents the average of all values collected by day.

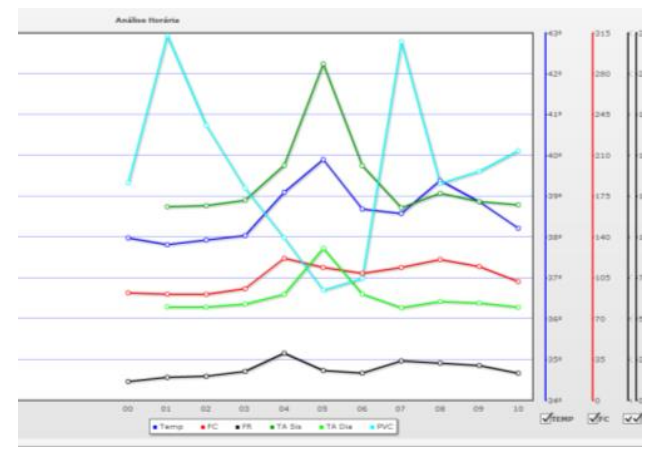

Fig. 3. Vital Signs Chart (Hour)

\subsection{Medical Scores}

This feature calculates automatically and in real time the most used medical scores in Intensive Medicine. This system allows obtaining the results of SAPS II, SAPS III, SOFA, GLASGOW, TISS28 and MEWS. This component is divided in three parts and it is an integrant part of ENR. The first component is used to validate the values automatically collected or to insert the values in fault - the interface is touch-screen and intuitive to use. The second part present a table with the results obtained since patient admission. The third part presents all the values in a new and interesting way. From some scores: SAPS, SOFA, GLASGOW and MEWS it is possible analyze the evolution of a patient in a way similar to the vital signs.

The system computes the scores whenever some new value arrives and presents the final score result in the chart, showing the evolution between the new result and the previous one. Figure 4 present an example of chart by minutes. In this case it is presenting the MEWS patient values.

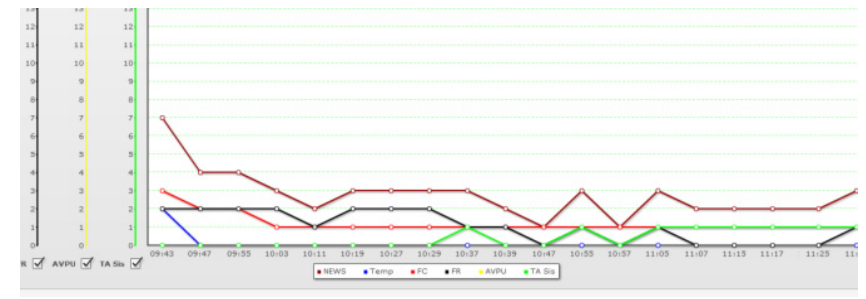

Fig. 4. MEWS Chart (Minutes) 


\subsection{Critical Events}

The introduction of critical events in ICU represents a novelty to this unit. Before this, nobody tracked the patient critical events, due the difficult to monitoring the patient in a continuous way. With the introduction of data streaming was possible tracking the patient critical events to five variables: Blood Pressure, Heart Rate, Spo2, Urine Output and Temperature. The tracking system is executed in real-time using automatic data acquisition and data processing tasks. The results obtained by the tracking system are presented inside of Electronic Nursing Record application. The results are presented in two different ways: a grid (table) and a chart. The table header is composed by 13 columns containing the number, the duration of each $\mathrm{CE}$ and the total of events. The table also has 24 lines, one for each hour of the day. The system fills the grid according to the patient values, i.e., if an event is critic or not and their duration. This way of visualizing events also has a warning system to alert if current values are out of range, i.e., if they are critical values.

These results are represented by a color system to alert to the patient condition. The charts present a new way of tracking the Critical Events. The user (doctor / nurse) can anywhere and anytime consult the evolution of a patient in regard to Critical Events. This type of consulting is available by minute, hour and day. Figure 5 makes an overview of chart for the minutes. In this figure is possible observe that the patient has a $\mathrm{SpO} 2$ critical event during the last 46 minutes. All the graphs are grouped by category (BP, SpO2, Temperature, Urine Output, and HR) and event type (1 or 2).

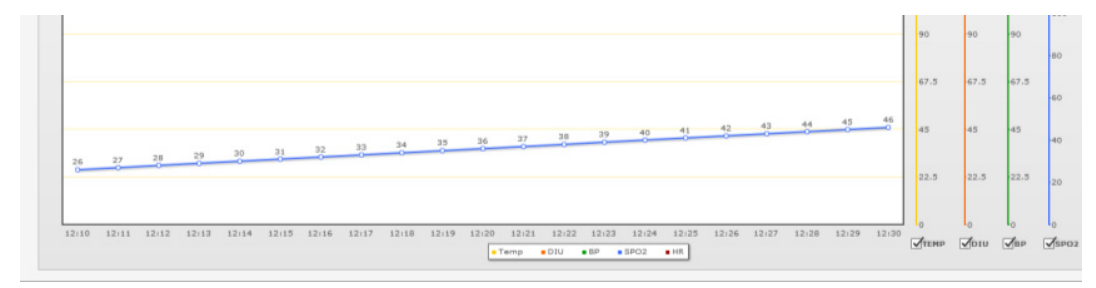

Fig. 5. Critical Events Chart by minute

\subsection{Ensemble Data Mining}

Using Data Mining it was possible induce an ensemble automatically and in real-time. This ensemble compares all the results obtained by each one of the models and chooses the best, i.e., select the model which meets the quality measure and present the best values. A set of experiments have been carried out in order to test the ensemble performance. The ensemble considered a set of Online and real-time data collected from the ICU. The data used to generate the DM models were gathered in the ICU of CHP - HSA during the period between February and June of 2012 and it is related to the first five days of stay of 129 patients. To evaluate the ensemble three measures were considered: Sensitivity, Accuracy, and Total Error (Terror). For each measure the average and the standard deviation of 10 runs was taken.

The selected models are used by the pervasive system only if they satisfy the following conditions: Total Error $<=40 \%$, Sensitivity $>=85 \%$ and Accuracy $>=60 \%$ 
These thresholds were defined in order to assure a minimum level of quality in models. The measure was defined in accordance with ICU doctors. The values can be adjustable anytime and are commonly accepted in the medical community.

Table 4 presents the performance achieved by the ensemble for each target. The values correspond to the average of the measures obtained during ten runs of the ensemble. For each value it is associated the standard deviation. The respiratory, hepatic and renal systems don't meet the measures established and aren't yet considered by the pervasive system.

Table 4. Ensemble Data mining primary Results

\begin{tabular}{lccccc}
\hline \multicolumn{1}{c}{ Target } & $\begin{array}{c}\text { Accepted by } \\
\text { quality measures }\end{array}$ & \multicolumn{1}{c}{ Sensitivity } & Accuracy & Specificity & Terror \\
\hline Cardiovascular & YES & $\mathbf{9 7 , 9 5} \pm 0,31$ & $\mathbf{7 6 , 8 1} \pm 2,35$ & $\mathbf{4 1 , 8 1} \pm 5,75$ & $\mathbf{2 3 , 1 9} \pm 2,35$ \\
Coagulation & YES & $\mathbf{9 1 , 2 0} \pm 3,57$ & $\mathbf{6 5 , 6 9} \pm 3,83$ & $\mathbf{4 9 , 6 1} \pm 6,15$ & $\mathbf{3 4 , 3 1} \pm 3,84$ \\
Hepatic & NO & $\mathbf{6 9 , 2 4} \pm 9,41$ & $\mathbf{8 2 , 8 9} \pm 2,57$ & $\mathbf{8 7 , 3 4} \pm 3,22$ & $\mathbf{1 7 , 1 0} \pm 2,57$ \\
Outcome & YES & $\mathbf{9 9 , 7 7} \pm 0,33$ & $\mathbf{6 3 , 5 8} \pm 3,11$ & $49,58 \pm 4,90$ & $\mathbf{3 6 , 4 2} \pm 3,11$ \\
Renal & NO & $\mathbf{7 7 , 1 7} \pm 12,41$ & $\mathbf{4 3 , 0 8} \pm 4,66$ & $\mathbf{4 3 , 0 8} \pm 4,66$ & $\mathbf{4 9 , 0 9} \pm 5,39$ \\
Respiratory & NO & $67,11 \pm 5,67$ & $\mathbf{6 3 , 8 6} \pm 4,27$ & $60,39 \pm 6,75$ & $36,14 \pm 4,27$ \\
\hline
\end{tabular}

Figure 6 makes an overview of the prevision system. This figures show which is the probability of occur an organ failure or patient die. For each type of target some different colors are used. In the case of organ failure the range is: $0 \%$ to $5 \%$ - green $6 \%$ to $49 \%$ - yellow; $50 \%$ to $69 \%$ - orange; $70 \%$ to $100 \%$ - red. At level of outcome the range is $0 \%$ to $5 \%$ - green; $5 \%$ to $49 \%$ - yellow; $50 \%$ to $85 \%$ orange; $86 \%$ to $100 \%$ - red. For example the probability of this patient die in the next hour is $78 \%$.

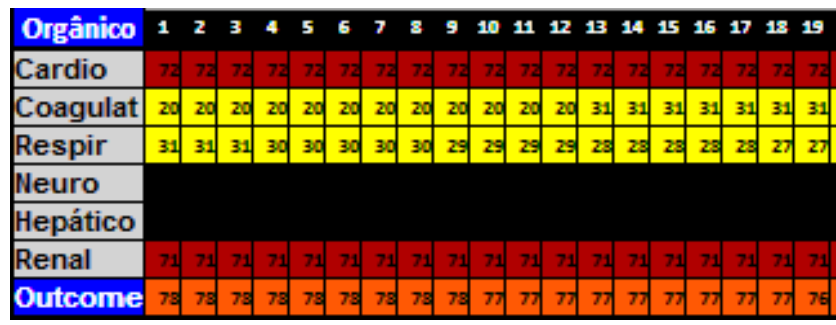

Fig. 6. INTCare prediction system

In order to assess the technology acceptance by the ICU professionals a set of questionnaires using Technology Acceptance Method (TAM) [24] were performed. This process had as main objective understand the system importance / quality and the user acceptance. A questionnaire was used to elaborate some queries about the decision process and the achievement of new knowledge. The questionnaire was answered by $1 / 3$ of ICU nurses and in a 5 points scale ( 1 - worst; 5 - excellent) had an average upper than 3 points. The questionnaires results [25] showed that the information generated (knowledge) and the system deployed are very important to the ICU professionals, being them suitable and framed in the decision making process. 


\section{CONCLUSIONS}

This paper presents evidences that it is possible to deploy a pervasive intelligent decision support system to support the decision making process in intensive medicine. The work carried out reaches an important milestone: to computerize all the data acquisition and data transforming processes in critical areas in order to discover new knowledge and adapt in real-time.

First of all it has been showed the importance of the preparation of the environment in order to support pervasive features. Then, a set of changes should be introduced in the information system and in the way of how the environment collects, processes and transforms the data. Finally, there are large benefits in using inference systems to automatically and in real-time receive the data, process them according to the targets and execute the tasks associated to the development of new knowledge.

One important feature for the ICU professionals (users) it is how the new knowledge is presented. Two platforms have been developed. One called Electronic Nursing Record (ENR) for monitoring the patient data (insert, edit and validate the values) and to present the results associated to the patient as is vital signs, critical events and medical scores. ENR presents a new way of evaluating the patient condition comparing the current results with those obtained previously. The second platform is focused in the presentation of the results provided by the Data Mining engine, i.e., the ensemble results and the probability of occurring an organ failure and outcome. Both systems are integrated in the ICU information system and can be viewed as a single system. These systems can be accessed by anyone who has the proper privileges. Automatic induction of Data Mining models can be considered the main contribution of this system allowing for predicting clinical events in real-time for the next hour. These models adapt and optimize over the time ensuring that the best predictions are presented whenever requested.

The system has been assessed using the TAM method. The results corroborated the importance of the system for the ICU professionals and motivate futures developments.

Acknowledgments. This work has been supported by FCT - Fundação para a Ciência e Tecnologia in the scope of the project: PEst-OE/EEI/UI0319/2014. The authors would like to thank FCT for the financial support through the contract PTDC/EIA/72819/ 2006 (INTCare) and PTDC/EEI-SII/1302/2012 (INTCare II). The work of Filipe Portela was supported by the grant SFRH/BD/70156/2010 from FCT.

\section{References}

1. Silva, Á., Cortez, P., Santos, M.F., Gomes, L., Neves, J.: Rating organ failure via adverse events using data mining in the intensive care unit. Artificial Intelligence in Medicine 43, 179-193 (2008)

2. Mador, R.L., Shaw, N.T.: The impact of a Critical Care Information System (CCIS) on time spent charting and in direct patient care by staff in the ICU: a review of the literature. International Journal of Medical Informatics 78, 435-445 (2009)

3. Gago, P., Santos, M.F., Silva, Á., Cortez, P., Neves, J., Gomes, L.: INTCare: a knowledge discovery based intelligent decision support system for intensive care medicine. Journal of Decision Systems (2006) 
4. Portela, F., Santos, M., Vilas-Boas, M., Rua, F., Silva, Á., Neves, J.: Real-time Intelligent decision support in intensive medicine. KMIS 2010- International Conference on Knowledge Management and Information Sharing, pp. 7, Valência, Espanha (2010)

5. Manuel Santos, Filipe Portela, Marta Vilas-Boas, José Machado, António Abelha, José Neves, Álvaro Silva, Fernando Rua, Maria Salazar, César Quintas, Cabral, A.: Intelligent Decision Support in Intensive Care Units Nursing Information Modelling. (2009)

6. Wooldridge, M.: Intelligent agents. Multiagent systems: a modern approach to distributed artificial intelligence, pp. 27-77. MIT Press (1999)

7. Portela, F., Santos, M.F., Silva, Á., Machado, J., Abelha, A.: Enabling a Pervasive approach for Intelligent Decision Support in Critical Health Care. In: publication, S.-A.t. (ed.) HCist 2011 International Workshop on Health and Social Care Information Systems and Technologies, pp. 10, Algarve, Portugal (2011)

8. Kohn, L.T., Corrigan, J., Donaldson, M.S.: To Err Is Human: Building a Safer Health System. National Academy Press (2000)

9. Varshney, U.: Pervasive Healthcare Computing: EMR/EHR, Wireless and Health Monitoring. Springer-Verlag New York Inc (2009)

10. Scicluna, P., Murray, A., Xiao, Y., Mackenzie, C.F.: Challenges to Real-Time Decision Support in Health Care. Agency for Healthcare Research and Quality (2008)

11. Kaur, M., Pawar, M., Kohli, J.K., Mishra, S.: Critical events in intensive care unit. Indian Journal of Critical Care Medicine: Peer-reviewed, Official Publication of Indian Society of Critical Care Medicine 12, 28 (2008)

12. Brenck, F., Hartmann, B., Mogk, M., Junger, A.: [Scoring systems for daily assessment in intensive care medicine. Overview, current possibilities and demands on new developments]. Der Anaesthesist 57,189 (2008)

13. Vincent, J.L., Bruzzi de Carvalho, F.: Severity of illness. Semin Respir Crit Care Med 31, 031-038 (2010)

14. Vincent, J.L., Moreno, R., Takala, J., Willatts, S., De Mendonca, A., Bruining, H., Reinhart, C.K., Suter, P.M., Thijs, L.G.: The SOFA (Sepsis-related Organ Failure Assessment) score to describe organ dysfunction/failure. Intensive care medicine 22, 707-710 (1996)

15. Jones, C.: Glasgow coma scale. AJN The American Journal of Nursing 79, 1551 (1979)

16. Reis Miranda, D., de Rijk, A., Schaufeli, W.: Simplified Therapeutic Intervention Scoring System: the TISS-28 items--results from a multicenter study. Critical care medicine 24, 64 (1996)

17. Gardner-Thorpe, J., Love, N., Wrightson, J., Walsh, S., Keeling, N.: The value of Modified Early Warning Score (MEWS) in surgical in-patients: a prospective observational study. Annals of The Royal College of Surgeons of England 88, 571 (2006)

18. Gaber, M.M., Zaslavsky, A., Krishnaswamy, S.: Mining data streams: a review. ACM Sigmod Record 34, 18-26 (2005)

19. Kantardzic, M.: Data mining: concepts, models, methods, and algorithms. Wiley-IEEE Press (2011)

20. Lee, P.: Design Research: What Is It and Why Do It? In: Reboot (ed.) The Reboot, vol. 2013, http://thereboot.org (2012)

21. Lunenfeld, P., Laurel, B.: Design research: Methods and perspectives. MIT press (2003)

22. Portela, F., Santos, M.F., Vilas-Boas, M.: A Pervasive Approach to a Real-Time Intelligent Decision Support System in Intensive Medicine. In: Springer (ed.) Communications in Computer and Information Science, vol. 0272, pp. 14 (2012)

23. Saha, D., Mukherjee, A.: Pervasive computing: a paradigm for the 21 st century. IEEE Computer 36 , 25-31 (2003)

24. Chooprayoon, V., Fung, C.C.: TECTAM: An Approach to Study Technology Acceptance Model (TAM) in Gaining Knowledge on the Adoption and Use of E-Commerce/E-Business Technology among Small and Medium Enterprises in Thailand. (2010)

25. Filipe Portela, Jorge Aguiar, Manuel Filipe Santos, Álvaro Silva, Fernado Rua: Pervasive Intelligent Decision Support System - Technology Acceptance in Intensive Care Units. In: Springer (ed.) Advances in Intelligent Systems and Computing. Springer (2013) 